\title{
Summary of: \\ The Kushida Index as a screening tool for obstructive sleep apnoea-hypopnoea syndrome
}

\author{
S. Jauhar, ${ }^{1}$ R. Orchardson, ${ }^{2}$ S. W. Banham, ${ }^{3}$ E. Livingston, ${ }^{4}$ A. Sherriff ${ }^{5}$ \\ and M. F. Lyons ${ }^{6}$
}

VERIFIABLE CPD PAPER

\begin{tabular}{|c|}
\hline FULL PAPER DETAILS \\
\hline 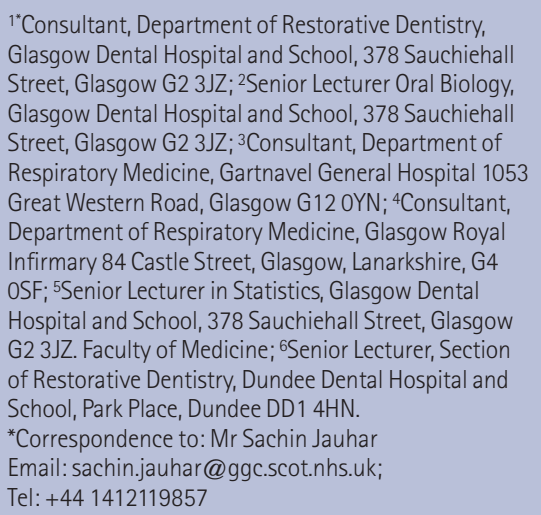 \\
\hline 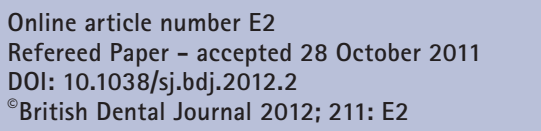 \\
\hline
\end{tabular}

\begin{abstract}
Objectives To test the validity of the Kushida Index for screening for sleep apnoea in a West of Scotland adult population. Methods Specific intra-oral measurements and respiratory polysomnography were carried out on 71 patients in this prospective study. The intra-oral measurements were applied to the Kushida formula to obtain a value for the Kushida Index. This value was compared to the diagnosis obtained using polysomnography in the conventional manner. Results The sensitivity of the Kushida Index in this present study was 68\% (95\% Cl 50-81) and the specificity was 71\% (95\% Cl 52-84). The positive predictive value was 71\% and the negative predictive value was 67\%. The Mallampati score, Epworth sleepiness score and enlargement of the tongue, soft palate or tonsils were not statistically significantly related to a diagnosis of sleep apnoea ( $p>0.05)$. Conclusion With the limited sensitivity and specificity of the Kushida Index demonstrated in this study, this test cannot be recommended as a screening tool for sleep apnoea in a West of Scotland population.
\end{abstract}

\section{EDITOR'S SUMMARY}

The diversity of activity within dental practices in recent years has been quite marked. Even a decade ago the likelihood of practices being involved in smoking cessation, Botox applications or treatment for snoring and sleep apnoea was small but has grown enormously. What has also needed to increase is the co-operation between dental team members but also between other dental and medical specialists in order to provide a joined-up service to patients as well as a continuity of professional monitoring and care.

In terms of the diagnosis of snoring and/or obstructive sleep apnoea-hypopnoea syndrome the borderline between what is possible and accurate in dental practice and what needs to be undertaken in collaboration with a specialist facility, in this case a sleep clinic, is quite crucial if the correct diagnosis is to be made. This study throws some doubt on the previous findings for the applicability of the Kushida Index in this context, although maintains the value that treatment in the dental context can provide.

This need for co-operative interdisciplinary working should not be a barrier to dentists becoming involved in wider aspects of healthcare and patient wellbeing. In fact, on the contrary, the ability to be able to offer a greater range of therapies is to the advantage of both the patient and the dentist, particularly for the latter in terms of professional satisfaction and greater engagement of knowledge, skill and experience. Indeed the challenge of learning more about the aetiology, pathology and management of conditions such as these provide an often welcome stimulus in a world that might otherwise have become routine and somewhat limited. It is, however, prudent to always work within one's limits and the boundaries of individual knowledge and competence. In this context the need for thorough research and appropriate support is of greater importance still and this study serves to further illuminate the extent of our current knowledge base while questioning its validity in certain vital aspects.

The full paper can be accessed from the $B D J$ website (www.bdj.co.uk), under 'Research' in the table of contents for Volume 212 issue 1.

Stephen Hancocks Editor-in-Chief

DOI: 10.1038/sj.bdj.2012.27 
TO ACCESS THE BDJ WEBSITE TO READ THE FULL PAPER:

- BDA Members should go to www.bda.org.

- Click the 'login' button on the right-hand side and enter your BDA login details.

- Once you have logged in click the 'BDJ' tab to transfer to the BDJ website with full access.

IF YOUR LOGIN DETAILS DO NOT WORK:

- Get a password reminder: go to www.bda.org, click the login button on the right-hand side and then click the forgotten password link.

- Use a recommended browser: we recommend Microsoft Internet Explorer or Mozilla Firefox.

- Ensure that the security settings on your browser are set to recommended levels.

IF YOU HAVE NOT YET SIGNED UP TO USE THE BDA WEBSITE:

- Go to www.bda.org/getstarted for information on how to start using the BDA website.
IN BRIEF

- Outlines the possible role of the dentist in screening for obstructive sleep apnoeahypopnoea syndrome (OSAHS).

- Assesses the validity of screening for OSAHS using the Kushida Index by dentists in the West of Scotland.

\section{AUTHOR QUESTIONS AND ANSWERS}

1. Why did you undertake this research? Dentists are in a good position to screen patients for OSAHS as they regularly see patients for routine examinations. The consequences of undiagnosed OSAHS are significant. This research was undertaken to assess if a dentist could use this previously established screening tool to accurately screen patients for OSAHS.

\section{What would you like to do next in this} area to follow on from this work?

The Kushida Index is a simple screening tool that dentists can use, but this study failed to corroborate it as a robust screening tool for OSAHS. If the tool was effective the next step would be to carry out a large scale primary care-based study, however, in view of the negative findings of the study the authors would suggest alternative methods of screening are used. All practitioners should work in liaison with a sleep specialist and refer as appropriate.
The dentist who chooses to help manage patients who snore is faced with the dilemma of knowing whether his/her patient has simple snoring or OAHS. Sleep studies are expensive, time consuming and inconvenient. It would be impossible and unnecessary for all individuals who snore to be investigated in this way.

Screening for disorders should ensure that at-risk individuals are identified and are offered further appropriate investigation and management.

The present study is an example of research collaboration between dentists and respiratory physicians and

\section{R. Jagger}

Consultant Senior Lecturer,

Department of Oral and Dental Sciences, University of Bristol

1. Dental Protection Ltd. Snoring and obstructive sleep apnoea syndrome. Position statement available online at http://www.dentalprotection.org/uk/news/positionstatements/snoring (accessed 19 December 2011).

2. Dental Defence Union. Personal communication 19 December 2011.

3. Stradling J, Dookun R. Snoring and the role of the GDP: British Society of Dental Sleep Medicine (BSDSM) pre-treatment screening protocol. Br Dent J 2009; 206: 307-312. 